\title{
Obstacles to Factorization of Partial Differential Operators into Several Factors
}

\author{
E. S. Shemyakova and F. Wincler \\ RISC-Linz, J. Kepler University Linz, A-4040 Linz, Austria \\ e-mail:kath@risc.uni-linz.ac.at; Franz.Winkler@risc.uni-linz.ac.at
}

Received June 4, 2006

\begin{abstract}
In the paper, construction of algorithms for factoring linear partial differential operators is studied. New theoretical concepts-obstacle and ring of obstacles - are introduced. These are invariants and posses other interesting properties. A theorem on unique factorization extension starting from some moment, which is important from the standpoint of construction of such algorithms, is proved. Obstacles in the case of the second and third degrees are found.
\end{abstract}

DOI: $10.1134 / \mathrm{S} 036176880702003 \mathrm{X}$

\section{INTRODUCTION}

We study the problem of factoring linear partial differential operators over some field. The study originates from the Grigoriev-Schwarz algorithm [1], which extends factorization (with mutually simple factors) of an operator symbol to the factorization of the operator itself. At the first step of the algorithm, only leading components of the factors are known. Then, at each subsequent step, one either finds the next component in each factor or proves that factorization of this type does not exist. In the latter case, all accumulated information about the operator is lost: at each step of the algorithm, a partial factorization of the operator is implicitly found. We suggest using this information.

In so doing, we arrive at the concepts of partial factorization and ordinary obstacle. In the case of the second-order operators, the latter coincides with the wellknown Laplace invariants [2].

The partial factorizations made it possible to prove Theorem 1 on unique extension of operator factorization starting from some moment. The GrigorievSchwarz theorem [1] is a particular case of this theorem.

Generally, neither ordinary obstacle nor its symbol is unique or invariant (some examples were considered in [3]). We introduce a new concept - a ring of obstacles-which is defined as a quotient ring of polynomials corresponding to the ring of linear differential operators modulo some uniform ideal. Symbols of all ordinary obstacles belong to the same adjacent class in this ring of obstacles. We named this class an obstacle to factorization. One important feature of an obstacle is that it is invariant with respect to the conjugation operation. We also prove some other interesting features.

Explicit formulas for obstacles of the second- and third-degree operators of two variables are found.
It should be noted that this work has already found an application: a complete system of invariants for a third-order hyperbolic operator of two variables has been derived [4].

This study is a sequel to work [5], where factorization into two factors has been considered.

\section{PRELIMINARY FACTS}

Let $K$ be a field with differentiation operations $D_{1}, \ldots, D_{n}$ defined in it. Consider a ring of linear differential operators $K[D]=K\left[D_{1}, \ldots, D_{n}\right]$. The set of all linear differential operators of order $\leq i$ equipped with the left and right multiplication is a $K$-bimodule denoted as $K_{\leq i}$. Thus, we have filtering $\ldots \supset K_{\leq i} \supset K_{\leq i-1} \supset \ldots \supset K_{\leq 0}$. Consider the adjoint algebra

$$
S m b l_{*}=\sum_{i \geq 0} S m b l_{i}, \quad S m b l_{i}=K_{\leq i} / K_{\leq i-1} .
$$

The $K$-module $S m b l_{*}$ is a commutative $K$-algebra isomorphic to the ring of polynomials $K[X]=K\left[X_{1}, \ldots\right.$, $\left.X_{n}\right]$ in $n$ variables. The image of the operator $L \in K[D]$ upon natural projection will be considered as an element of the ring of polynomials $K[X]$ and denoted as $\mathrm{Sym}_{L}$. In fact, an operator symbol is a homogeneous polynomial corresponding to the sum of leading terms of the operator.

We use the following notation:

$$
\begin{gathered}
D^{\left(i_{1}, \ldots, i_{n}\right)}:=D_{1}^{i_{1}} \ldots D_{n}^{i_{n}}, \\
\left|D^{\left(i_{1}, \ldots, i_{n}\right)}\right|=\operatorname{ord}\left(D^{\left(i_{1}, \ldots, i_{n}\right)}\right):=i_{1}+\ldots+i_{n} .
\end{gathered}
$$

By definition, the order of the zero operator is $-\infty$. For a homogeneous polynomial $S \in K[X]$, the operator obtained by substitution of $D_{i}$ for $X_{i}$ is denoted as $\hat{S} \in$ 
$K[D]$. Both the polynomial and the corresponding operator are denoted by one letter if this does not result in any confusion. The set of all operators from $K[D]$ of order $i$ is denoted as $K_{i}[D]$. form

Any operator $L \in K[D]$ of order $d$ is written in the

$$
L=\sum_{|J| \leq d} a_{J} X^{J}=\sum_{i=0}^{d} L_{i},
$$

where $a_{J} \in K, J \in \mathbf{N}^{n}$, and $L_{i}$ is the component of $L$ of order $i$.

\section{PARTIAL FACTORIZATIONS}

We begin with several definitions presented in [5] for the case of two factors.

Definition 1. Let $L \in K[D]$ and $\operatorname{Sym}_{L}=S_{1} \ldots S_{k}$. The factorization $L=F_{1} \circ \ldots \circ F_{k}$ is said to have type $\left(S_{1}\right)\left(S_{2}\right) \ldots\left(S_{k}\right)$ if $\operatorname{Sym}_{F_{i}}=S_{i} \forall i \in\{1, \ldots, k\}$.

Definition 2. Let, for some operators $L, F_{i} \in K[D]$, $i=1, \ldots, k$, and, for some $t \in\{0, \ldots, \operatorname{ord}(L)\}$,

$$
\operatorname{ord}\left(L-F_{1} \circ \ldots \circ F_{k}\right)<t \text {. }
$$

Then, $F_{1} \circ \ldots \circ F_{k}$ is called a partial factorization of operator $L$ of order $t$. If, additionally, $S_{i}=\operatorname{Sym}_{F_{i}}, i=$ $1, \ldots, k$ (and, hence, $\operatorname{Sym}_{L}=S_{1} \ldots S_{k}$ ), then the partial factorization has type $\left(S_{1}\right) \ldots\left(S_{k}\right)$.

Remark 1. Any factorization (in ordinary sense) $L \in K[D]$ is a partial factorization of order 0 .

Remark 2. Let $L \in K[D]$, ord $(L)=d$. Then, for any factorization of the symbol $\operatorname{Sym}_{L}=S_{1} \ldots S_{k}$, the corresponding operator composition $\hat{S}_{1} \circ \ldots \circ \hat{S}_{k}$ is a partial factorization of order $d$.

Suppose that $L \in K[D]$ and $F_{1} \circ \ldots \circ F_{k}$ is its partial factorization of order $t$. It is not difficult to see that condition (2) holds independent of what terms of order $<t-$ $\left(d-d_{i}\right)$ are selected in the factor $F_{i}$. Hence, we obtain new partial factorizations of order equal to or less than $t$. Therefore, it is natural to introduce the following definition.

Definition 3. Let $L \in K[D], \operatorname{Sym}_{L}=S_{1} \ldots S_{k}$, $\operatorname{ord}\left(S_{i}\right)=d_{i}, i=1, \ldots, k$, and

$$
F_{1} \circ \ldots \circ F_{k}, \quad F_{1}^{\prime} \circ \ldots \circ F_{k}^{\prime}
$$

be partial factorizations of orders $t$ and $t^{\prime}$, respectively. Let $t^{\prime}<t$. Then, $F_{1}^{\prime} \circ \ldots \circ F_{k}^{\prime}$ extends $F_{1} \circ \ldots \circ F_{k}$ if

$$
\operatorname{ord}\left(F_{i}-F_{i}^{\prime}\right)<t-\left(d-d_{i}\right), \quad \forall i \in\{1, \ldots, k\} .
$$

Example 1. Consider the fifth-order operator

$L=\left(D_{1}^{2}+D_{2}+1\right) \circ\left(D_{1}^{2} D_{2}+D_{1} D_{2}+D_{1}+1\right)$.

Factorizations of the form

$$
\left(D_{1}^{2}+\ldots\right) \circ\left(D_{1}^{2} D_{2}+\ldots\right),
$$

where ellipses denote arbitrarily selected terms of lower orders, are partial factorizations of order five for which the fourth-order extensions are partial factorizations of the form

$$
\left(D_{1}^{2}+D_{2}+\ldots\right) \circ\left(D_{1}^{2} D_{2}+D_{1} D_{2}+\ldots\right) .
$$

Remark 3. Let $L \in K[D]$. Then, $F_{1} \circ \ldots \circ F_{k}$ is its partial factorization of form $\left(S_{1}\right) \ldots\left(S_{k}\right)$ if and only if $F_{1} \circ \ldots \circ F_{k}$ is an extension of the partial factorization $S_{1} \circ \ldots \circ S_{2}$.

The following two propositions can easily be proved and will be used in proving the theorem below.

Proposition 1. Let $S_{1}, S_{2}$, and $p$ be homogeneous polynomials in an arbitrary number of independent variables of degrees $d_{1}, d_{2}$, and $s\left(0<s<d_{1}+d_{2}\right)$, respectively. Let $S_{1}$ and $S_{2}$ be mutually simple. Then, there does not exist more than one pair $(u, v)$ of homogeneous polynomials $u$ and $v$ of degrees $s-d_{1}$ and $s-$ $d_{2}$, respectively, such that

$$
S_{1} \cdot u+S_{2} \cdot v=p .
$$

The second assertion is an extension of Proposition 1 to the case of polynomials that are not mutually simple.

Proposition 2. Let $S_{1}, S_{2}$, and $p$ be homogeneous polynomials in an arbitrary number of variables of degrees $d_{1}, d_{2}$, and $s$, respectively. Let polynomial $S_{0}$ of degree $d_{0}$ be the greatest common divisor of $S_{1}$ and $S_{2}$, and let $0<s<d_{1}+d_{2}-d_{0}$. Then, there does not exist more than one pair $(u, v)$ of homogeneous polynomials $u$ and $v$ of degrees $s-d_{1}$ and $s-d_{2}$, respectively, such that

$$
S_{1} \cdot u+S_{2} \cdot v=p .
$$

For any factorization $S_{1} \cdot S_{2}$ of a symbol, the corresponding composition of the operators $\hat{S}_{1} \circ \hat{S}_{2}$ is a partial factorization of the operator $L$. If $S_{1}$ and $S_{2}$ are mutually simple, there does not exist more that one extension of this partial factorization to the factorization of $L$ [1]. If there exists a nontrivial common divisor of $S_{1}$ and $S_{2}$, this is not always true. For example, consider the Blumberg-Landau operator [6]

$$
\begin{aligned}
L=D_{x}^{3}+ & x D_{x}^{2} D_{y}+2 D_{x}^{2}+(2 x+2) D_{x} D_{y} \\
& +D_{x}+(2+x) D_{y} .
\end{aligned}
$$

This is an example of the operator that has two different factorizations into different number of factors (of course, the factors are irreducible, i.e., cannot be factored into lower-order factors):

$$
\begin{gathered}
L=\left(D_{x}+1\right) \circ\left(D_{x}+1\right) \circ\left(D_{x}+x D_{y}\right) \\
=\left(D_{x}^{2}+x D_{x} D_{y}+D_{x}+(2+x) D_{y}\right) \circ\left(D_{x}+1\right) .
\end{gathered}
$$


For the same operator $L$ (by the way, its symbol is $X^{3}+x X^{2} Y$ ), there exists a family of factorizations into two factors with the symbols $S_{1}=X$ and $S_{2}=X(X+X Y)$ :

$$
\begin{gathered}
L=\left(D_{x}+1+\frac{1}{x+f_{1}(y)}\right) \circ\left(D_{x}^{2}+x D_{x} D_{y}\right. \\
\left.+\left(1-\frac{1}{x+f_{1}(y)}\right) D_{x}+\left(x+1-\frac{x}{x+f_{1}(y)}\right) D_{y}\right),
\end{gathered}
$$

where $f_{1}(y) \in K$ is a functional parameter. Thus, the factorization is not unique. Nevertheless, certain results can be established even in this case where the factors are not mutually simple.

Theorem 1. Let $L \in K[D], \operatorname{Sym}_{L}=S_{1} \cdot S_{2}, \operatorname{ord}(L)=$ $d$, and polynomial $S_{0}$ of degree $d_{0}$ be the greatest common divisor of $S_{1}$ and $S_{2}$. Then, for any partial factorization of order $\left(d-d_{0}\right)$ of type $\left(S_{1}\right)\left(S_{2}\right)$, there does not exist more than one extension to the factorization of the operator $L$ of the same type.

The proof is based on the following lemma.

Lemma 1. Let $L \in K[D], \operatorname{Sym}_{L}=S_{1} \cdot S_{2}, \operatorname{ord}(L)=d$, and polynomial $S_{0}$ of degree $d_{0}$ be the greatest common divisor of $S_{1}$ and $S_{2}$. Then, for any partial factorization of type $t \leq\left(d-d_{0}\right)$ and order $t$, where $\left(S_{1}\right)\left(S_{2}\right)$, there does not exist more than one extension to the partial factorization of order $t-1$ (up to lower-order terms).

Proof. If $d_{0}=0$, the lemma assertion follows from results of [1]. If $d_{0}>0$, let us write the extension of a given factorization of order $t$ to the operator factorization in the general form as

$$
L=\left(\hat{S}_{1}+\sum_{j=0}^{k_{1}-1} G_{j}\right) \circ\left(\hat{S}_{2}+\sum_{j=0}^{k_{2}-1} H_{j}\right),
$$

where $k_{1}=\operatorname{ord}\left(S_{1}\right), k_{2}=\operatorname{ord}\left(S_{2}\right), G_{j} \in K_{j}[D], H_{i} \in K_{i}[D]$, $j=0, \ldots,\left(k_{1}-1\right), i=0, \ldots,\left(k_{2}-1\right)$. Comparing coefficients of terms of power $t-1$ on both sides of (4), we obtain

$$
L_{t-1}=H_{t-k_{1}-1} \cdot S_{1}+G_{t-k_{2}-1} \cdot S_{2}+P_{t-1},
$$

where $P_{t-1}$ is a homogeneous polynomial of degree $t$ that is uniquely computed by homogeneous polynomials $G_{i}$ and $H_{j}, i>t-k_{1}-1, j>t-k_{2}-1$ (components of the original partial factorization of order $t$ ). For $i<0$, the polynomials $G_{i}$ and $H_{i}$ are assumed equal to zero.

Now, since $t-1<d-d_{0}$, we may apply Proposition 2 , which says that there does not exist more than one solution to Eq. (5). That is, there does not exist more than one extension to the partial factorization of order $t-1$.

Corollary 1. If $S_{1}$ and $S_{2}$ are mutually simple, there does not exist more than one factorization of $L$ of type $\left(S_{1}\right)\left(S_{2}\right)$. That is, the Grigoriev-Schwarz theorem [1] is a particular case of the above theorem.

Corollary 2. For ordinary differential operators,

$$
\operatorname{GCD}\left(S_{1}, S_{2}\right)=X^{d_{0}}
$$

where

$$
d_{0}=\min \left(\operatorname{ord}\left(S_{1}\right), \operatorname{ord}\left(S_{2}\right)\right) .
$$

Then, for any partial factorization of order

$$
\max \left(\operatorname{ord}\left(S_{1}\right), \operatorname{ord}\left(S_{2}\right)\right)-1,
$$

there does not exist more than one extension to an ordinary factorization.

Corollary 3. Let $L \in K[D], \operatorname{Sym}_{L}=S_{1} \cdot S_{2}$, and $S_{1}$ and $S_{2}$ be mutually simple. Then, for any $t, t<\operatorname{ord}(L)$, there does not exist more than one (up to lower-order terms) partial factorization of order $t$.

\section{RING OF OBSTACLES, OBSTACLES}

The following theorem is proved by induction on the number of factors with the use of the theorem on uniqueness of factorization in the case of two factors [1].

Theorem 2. Let $L \in K[D], \operatorname{Sym}_{L}=S_{1} \cdot S_{2} \ldots S_{k}$, and $S_{1}, \ldots, S_{k}$ be mutually simple. Then, there does not exist more than one factorization of type $\left(S_{1}\right)\left(S_{2}\right) \ldots\left(S_{k}\right)$.

Consider factorable operators as a submanifold of all operators from $K[D]$ with a given symbol factorization.

Theorem 3. Let $K$ be a field. Consider the submanifold of operators from $K[D]$ with the fixed symbol $\operatorname{Sym}=S_{1} \ldots S_{k}, \operatorname{ord}\left(S_{i}\right)=d_{i}, i=1, \ldots, k$. Then, the codimension of the submanifold of operators having factorization of type $\left(S_{1}\right)\left(S_{2}\right) \ldots\left(S_{k}\right)$ is

$$
\left(\begin{array}{c}
n+d-1 \\
n
\end{array}\right)-\sum_{i=1}^{k}\left(\begin{array}{c}
n+d_{i}-1 \\
n
\end{array}\right) .
$$

Proof. Any operator $L$ from the considered set can be written in the form

$$
L=\left(S_{1}+\sum_{i=0}^{d_{1}-1} G_{i}^{1}\right) \circ \ldots \circ\left(S_{k}+\sum_{i=0}^{d_{k}-1} G_{i}^{k}\right),
$$

where $G_{i}^{j}$ denotes the $i$ th power component in the $j$ th factor. Comparing components of power $t, 0 \leq t \leq$ $\operatorname{ord}(L)-1$, on both sides of (6), we obtain

$$
\begin{aligned}
P_{t}= & \left(\mathrm{Sym} / S_{1}\right) \cdot G_{t-d+d_{1}}^{1}+\ldots \\
& +\left(\mathrm{Sym} / S_{k}\right) \cdot G_{t-d+d_{k}}^{k},
\end{aligned}
$$

where $P_{t}$ is a homogeneous polynomial of degree $t$ uniquely computable by the homogeneous polynomials $G_{i}, H_{j}, i>t-k_{1}, j>t-k_{2}$. Hence, if the equation is solved in the "decreasing" order (i.e., starting from $t=$ $\operatorname{ord}(L)-1$ with $t$ decreasing by one at each step), this polynomial can be considered known.

The polynomials $G_{i}, H_{j}, i>t-k_{1}, j>t-k_{2}$, and, hence, $P_{t}$ are determined uniquely, which immediately follows from Lemma 2. 
Lemma 2. Let $S_{1}, \ldots, S_{k}$ be mutually simple homogeneous polynomials of degrees $d_{1}, \ldots, d_{k}$, respectively. Let us denote $S=S_{1} \ldots S_{k}$. Then, there does not exist more than one set $\left(A_{1}, \ldots, A_{k}\right)$ such that

$$
P_{t}=\left(S / S_{1}\right) \cdot A_{1}+\ldots+\left(S / S_{k}\right) \cdot A_{k},
$$

where $\operatorname{ord}\left(P_{t}\right)=t, t<\operatorname{ord}(S)$, and $\operatorname{ord}\left(A_{i}\right)+\operatorname{ord}\left(S / S_{i}\right)=t$.

Proof. Suppose that there exist two such sets, $\left(A_{1}^{\prime}\right.$, $\left.\ldots, A_{k}^{\prime}\right)$ and $\left(A_{1}^{\prime \prime}, \ldots, A_{k}^{\prime \prime}\right)$. Subtracting the equations corresponding to them, we obtain

$$
0=\left(S / S_{1}\right) \cdot B_{1}+\ldots+\left(S / S_{k}\right) \cdot B_{k},
$$

where $B_{i}=A_{i}^{\prime}-A_{i}^{\prime \prime}, i=1, \ldots, k$. Without loss of generality, we may assume that $B_{1} \neq 0$ and rewrite Eq. (9) as

$$
-\left(S / S_{1}\right) \cdot B_{1}=\left(S / S_{2}\right) \cdot B_{2}+\ldots+\left(S / S_{k}\right) \cdot B_{k} \text {. }
$$

Now, all addends on the right-hand side of the equation are divided by $S_{1}$, whereas $\left(S / S_{1}\right)$ is not divided by $S_{1}$. Hence, $S_{1}$ must divide $B_{1}$, and, thus, $\operatorname{ord}\left(B_{1}\right) \geq$ $\operatorname{ord}\left(S_{1}\right)$.

On the other hand, we know that $\operatorname{ord}\left(A_{i}\right)+$ $\operatorname{ord}\left(S / S_{i}\right)=t$ and $t<\operatorname{ord}(S)$; i.e., $\operatorname{ord}\left(A_{i}\right)<\operatorname{ord}\left(S_{i}\right)$, and, hence, $\operatorname{ord}\left(B_{1}\right)<\operatorname{ord}\left(S_{i}\right)$. This contradicts the conclusion of the previous paragraph and proves the lemma.

The existence of a factorization is determined by compatibility of the system of all equations (7), $t=d-$ $1, \ldots, 0$. The desired co-dimension is equal to the number of independent equations in the operator coefficients.

For each $t$, we have linear equation (7) in the polynomials $G_{t-d+d_{1}}^{1}, \ldots, G_{t-d+d_{k}}^{k}$, which is equivalent to a system of linear equations in their coefficients, $A \cdot \vec{g}=$ $\vec{c}$, where $A$ is the system matrix. Since the system has a unique solution, the rank of matrix $A$ is equal to the number of variables $V$; i.e., the columns of $A$ are linearly independent.

The system $A \cdot \vec{g}=\vec{c}$ is compatible when vector $\vec{c}$ belongs to the $V$-dimensional affine space spanned by the columns of $A$. The length of vector $\vec{c}$ is equal to the number of equations in the system. That is, the codimension of the space of solutions is equal to the difference of the number of the equations and the number of variables. The total co-dimension of the operators that have factorizations of the type $\left(S_{1}\right)\left(S_{2}\right) \ldots\left(S_{k}\right)$ is equal to the difference of the number of the equations and the number of variables in all steps altogether, which is found by using the well-known fact from the combinatorial analysis formulated in the following lemma.

Lemma 3. The cardinality of the set

$$
\left\{M=x_{1}^{d_{1}} \ldots x_{n}^{d_{n}} \mid d_{1}+\ldots+d_{n}=t\right\}
$$

of monomials in $n$ independent variables $x_{1}, \ldots, x_{n}$ is given by $\left(\begin{array}{c}n+t-1 \\ t\end{array}\right)=\left(\begin{array}{c}n+t-1 \\ n-1\end{array}\right)$.

The theorem on the co-dimension is proved.

Example 2. Consider all second-order operators of two independent variables with the symbol $S_{1} \cdot S_{2}$, where $S_{1}$ and $S_{2}$ are mutually simple fixed homogeneous operators of the first order. By Theorem 3, the codimension of the manifold of operators having factorizations of the type $\left(S_{1}\right)\left(S_{2}\right)$ is equal to 1 .

It is not difficult to find an explicit equation governing this manifold. For example, let $\hat{S}_{1}=D_{1}$ and $\hat{S}_{2}=$ $D_{2}$. Consider all operators of the form $L=D_{1} D_{2}+$ $a_{10} D_{1}+a_{01} D_{2}+a_{00}$. Such an operator has a factorization of type $\left(S_{1}\right)\left(S_{2}\right)$ if and only if the coefficients $a_{10}$, $a_{01}$, and $a_{00}$ satisfy the equation

$$
a_{00}-a_{10} a_{01}-\partial_{x}\left(a_{10}\right)=0 .
$$

Example 3. Consider all third-order operators of two independent variables with the symbol $S_{1} \cdot S_{2}$, where $S_{1}$ and $S_{2}$ are mutually simple fixed homogeneous operators of the first and second order, respectively. The co-dimension of the manifold of operators having factorizations of the type $\left(S_{1}\right)\left(S_{2}\right)$ is equal to 2 .

On the other hand, if we consider a factorization of the type $\left(S_{1}\right)\left(S_{2}\right)\left(S_{3}\right)$, where $S_{1}, S_{2}$, and $S_{3}$ are mutually simple fixed operators of the first order, the co-dimension is equal to 3 by Theorem 3 .

The following definition is needed for studying operators that have no factorizations of some types.

Definition 4. Let $L \in K[D]$ and $\operatorname{Sym}_{L}=S_{1} \ldots S_{k}$. An operator $R \in K[D]$ is said to be ordinary obstacle to factorization of $L$ of type $\left(S_{1}\right)\left(S_{2}\right) \ldots\left(S_{k}\right)$ if the operator $L-$ $R$ has factorization of type $\left(S_{1}\right)\left(S_{2}\right) \ldots\left(S_{k}\right)$ and the order of $R$ is minimal over all operators possessing this property.

Ordinary obstacles are closely related to partial factorizations introduced earlier.

Proposition 3. Let $L \in K[D]$ and $\operatorname{Sym}_{L}=S_{1} \ldots S_{k}$. Then, an ordinary obstacle to factorization of type $\left(S_{1}\right) \ldots\left(S_{k}\right)$ has order $t$ if and only if $t+1$ is the minimal order of partial factorization.

Clearly, ordinary obstacles are not uniquely determined. Moreover, their symbols are not uniquely determined as well. To get a unique (in a sense, invariant) concept, we introduce the following definition.

Definition 5. Let $L \in K[D]$ and $\operatorname{Sym}_{L}=S_{1} \cdot S_{2} \cdot \ldots \cdot S_{k}$. A ring of obstacles for factorization of type $\left(S_{1}\right) \ldots\left(S_{k}\right)$ is the quotient ring

$$
K\left(S_{1}, \ldots, S_{k}\right)=K[X] / I,
$$

where $I=\left(\frac{\operatorname{Sym}_{L}}{S_{1}}, \frac{\operatorname{Sym}_{L}}{S_{2}}, \ldots, \frac{\operatorname{Sym}_{L}}{S_{k}}\right)$ is a uniform ideal. 
Remark 4. In the case of two factors $(k=2)$, the ring of obstacles is given by

$$
K\left(S_{1}, S_{2}\right)=K[X] /\left(S_{1}, S_{2}\right),
$$

which coincides with the definition given in [5], where the case of two factors is considered.

Theorem 4. Let $L \in K[D]$ and $\operatorname{Sym}_{L}=S_{1} \cdot S_{2} \cdot \ldots$. $S_{k}$, where $S_{i}, i \in\{1, \ldots, k\}$, are mutually simple. Then, symbols of all ordinary obstacles of type $\left(S_{1}\right) \ldots\left(S_{k}\right)$ belong to the same adjacent class of the quotient ring $K\left(S_{1}, \ldots, S_{k}\right)$.

Proof. Let us denote $d_{i}=\operatorname{ord}\left(S_{i}\right), i \in\{1, \ldots, k\}$, and let $t$ be the order of ordinary obstacles. Repeating arguments from the proof of Theorem 3, we obtain Eq. (7); i.e., the symbol of any general obstacle can be written as

$$
\begin{aligned}
P_{t} & -\left(\left(\operatorname{Sym}_{L} / S_{1}\right) \cdot G_{t-d+d_{1}}^{1}+\ldots\right. \\
& \left.+\left(\operatorname{Sym}_{L} / S_{k}\right) \cdot G_{t-d+d_{k}}^{k}\right),
\end{aligned}
$$

where $P_{t}$ is a known uniquely determined polynomial, which is the same for all ordinary obstacles. That is, all ordinary obstacles belong to the adjacent class $\left[P_{t}\right]$ of the quotient ring $K\left(S_{1}, \ldots, S_{k}\right)$.

Definition 6. The adjacent class of symbols of ordinary obstacles in the ring of obstacles is called obstacle to factorization.

Remark 5. Any element from this adjacent class is an ordinary obstacle.

Definition 7. Factorization types $\left(S_{1}\right) \ldots\left(S_{k}\right)$ and $\left(b_{1} S_{1}\right) \ldots\left(b_{k} S_{k}\right)$ are similar if $b_{1}, \ldots, b_{k} \in K$ and $b_{1} \ldots b_{k}=1$.

Theorem 5. For a fixed operator, rings of obstacles and obstacles of similar types coincide.

Proof. Let us fix an operator $L \in K[D]$ and two similar factorization types, $\left(S_{1}\right) \ldots\left(S_{k}\right)$ and $\left(b_{1} S_{1}\right) \ldots\left(b_{k} S_{k}\right)$, where $b_{i} \in K, i=1, \ldots, k$. Then, the uniform ideals $\left(S_{1}\right.$, $\left.\ldots, S_{k}\right)$ and $\left(b_{1} S_{1}, \ldots, b_{k} S_{k}\right)$, and, hence, the rings of obstacles, coincide.

Any ordinary obstacle of type $\left(S_{1}\right) \ldots\left(S_{k}\right)$ and order $d_{0}$ can be written as

$$
P=L-\left(\hat{S}_{1}+T_{1}\right) \circ \ldots \circ\left(\hat{S}_{k}+T_{k}\right),
$$

where $T_{i}$ is the sum of components of orders $d_{i}-1, \ldots$, $d-d_{i}-d_{0}+1$ and $\operatorname{ord}(P)=d_{0}$.

There exist $T_{1}^{\prime}, \ldots, T_{k}^{\prime}$ such that $T_{i}^{\prime}$, like $T_{i}$, is the sum of components of orders $d_{i}-1, \ldots, d-d_{i}-d_{0}+1$, and

$$
\begin{gathered}
\left(S_{1}+T_{1}\right) \circ \ldots \circ\left(S_{k}+T_{k}\right) \\
=\left(b_{1} S_{1}+T_{1}^{\prime}\right) \circ \ldots \circ\left(b_{k} S_{k}+T_{k}^{\prime}\right) .
\end{gathered}
$$

Therefore, $P$ is ordinary obstacle of order $d_{0}$ of type $\left(b_{1} S_{1}\right) \ldots\left(b_{k} S_{k}\right)$. On the other hand, we have already proved that the obstacle rings $K\left(S_{1}, \ldots, S_{k}\right)$ and $\left(b_{1} S_{1}\right.$, $\ldots, b_{k} S_{k}$ ) coincide. Hence, the adjacent classes coincide as well; i.e., obstacles of similar types coincide.
Remind the following definition:

Definition 8. Let $g \in K^{*}$ be an invertible element from a ring $K$ and $L \in K[D]$. Then, the operator $g^{-1} \circ L \circ g$ is called conjugate of $L$.

Theorem 6. Ordinary obstacles for conjugate operators are conjugate: if $P$ is an ordinary obstacle for $L \in$ $K[D]$, then $g^{-1} P g$ is an ordinary obstacle for $g^{-1} L g$, where $g \in K^{*}$.

Proof. Let us consider an ordinary obstacle for $L$ of order $d_{0}$ given by (10). Let us find the conjugate obstacle using $g$ :

$$
\begin{gathered}
g^{-1} P g=g^{-1} L g-g^{-1} \circ\left(S_{1}+T_{1}\right) \\
\circ \prod_{j=2}^{k-1}\left(S_{i}+T_{i}\right) \circ\left(S_{k}+T_{k}\right) \circ g .
\end{gathered}
$$

There exist $T_{1}^{\prime}, \ldots, T_{k}^{\prime}$ such that $T_{i}^{\prime}$, like $T_{i}$, is the sum of components of orders $d_{i}-1, \ldots, d-d_{i}-d_{0}+1$, and

$$
\begin{gathered}
g^{-1} P g=g^{-1} L g-\left(g^{-1} S_{1}+T_{1}^{\prime}\right) \\
\circ \prod_{j=2}^{k-1}\left(S_{i}+T_{i}^{\prime}\right) \circ\left(g S_{k}+T_{k}^{\prime}\right) .
\end{gathered}
$$

Corollary 4. Obstacles to conjugate operators coincide.

Proof. Ordinary obstacles to conjugate operators are conjugate; hence, the symbol of ordinary obstacles is not changed.

Theorem 7. Let $n=2, L \in K[D]$, ord $(L)=d$, and $\operatorname{Sym}_{L}=S_{1} \ldots S_{k}$, where $S_{i}, i \in\{1, \ldots, k\}$, are mutually simple. Then, the obstacle ring $K\left(S_{1}, \ldots, S_{k}\right)$ raised to power $d-1$ is equal to zero (i.e., actual obstacles can be of order $d-2$ or less).

Proof. Let us denote $d_{i}=\operatorname{ord}\left(S_{i}\right), i \in\{1, \ldots, k\}$. Repeating arguments from the proof of Theorem 3, we obtain Eq. (7) for $t=d-1$ :

$$
P_{d-1}=\left(\operatorname{Sym}_{L} / S_{1}\right) \cdot G_{d_{1}-1}^{1}+\ldots+\left(\operatorname{Sym}_{L} / S_{k}\right) \cdot G_{d_{k}-1}^{k},
$$

which has at most one solution with respect to $G_{d_{1}-1}^{1}, \ldots, G_{d_{k}-1}^{k}$. Let us consider the corresponding system of linear equations in their coefficients. By Lemma 3, the number of equations in such a system is equal to $d$, and the number of variables is also equal to $d$, which means that the system has a unique solution. Hence, we have found a partial factorization of order $d-1$.

We remind that an operator $L \in K[D], \operatorname{ord}(L)=d$, is called strictly hyperbolic if its symbol is factored into $d$ different factors.

Theorem 8. Let $n=2$ and $L \in K[D]$ be a strictly hyperbolic operator of order $d$. Then, an ordinary obsta- 
cle of a fixed type to factorization into first-order factors is determined uniquely.

Proof. Let $\left(S_{1}\right) \ldots\left(S_{d}\right)$ be some factorization type and $P$ be an ordinary obstacle for this factorization type. Let the order of obstacles be $p$. Suppose that there is another ordinary obstacle of this type. Then, it can be written in the form

$$
P+\left(\operatorname{Sym}_{L} / S_{1}\right) \cdot A_{1}+\ldots+\left(\operatorname{Sym}_{L} / S_{d}\right) \cdot A_{d},
$$

where $A_{i}$ are homogeneous polynomials of degrees $p_{i}=$ $p-\operatorname{ord}\left(\operatorname{Sym}_{L} / S_{i}\right)=p-(d-1)$; i.e., $p \geq d-1$. On the other hand, by Theorem 7 , the ring of obstacles raised to power $d-1$ is equal to zero; therefore, $p \leq d-2$.

\section{SECOND-ORDER OPERATORS OF TWO VARIABLES}

Consider a second-order hyperbolic operator $L \in$ $K\left[D_{x}, D_{y}\right]$ in the coordinate system where its symbol has the form $X Y$. Then, by Theorems 7 and 8, both ordinary obstacles to this operator have order 0 and are uniquely determined. Let us find explicit formulas for them.

Theorem 9. Let

$$
L=D_{x} \cdot D_{y}+a D_{x}+b D_{y}+c,
$$

where $a_{10}, a_{01}, a_{00} \in K$. Then, obstacles of types $(X)(Y)$ and $(Y)(X)$ are given by

$$
\begin{aligned}
& c-a b-\partial_{x}(a), \\
& c-a b-\partial_{y}(b),
\end{aligned}
$$

respectively.

Proof. The factorization of $L$ of type $(X)(Y)$ has the form

$$
L=\left(D_{x}+g_{00}\right) \circ\left(D_{y}+h_{00}\right),
$$

where $g_{00}$ and $h_{00}$ are some elements of $K$. Comparing the first-order components on the left-hand and righthand sides of the equation, we obtain

$$
\left(a-h_{00}\right) D_{x}+\left(b-g_{00}\right) D_{y}=0 ;
$$

i.e., $a=h_{00}$ and $b=g_{00}$. Now, we calculate the obstacle as

$$
L-\left(D_{x}+b\right) \circ\left(D_{y}+a\right)=c-a b-\partial_{x}(a) .
$$

The obstacle of type $(Y)(X)$ is found similarly.

Remark 6. The obstacles found coincide with the Laplace invariants [2].

\section{THIRD-ORDER OPERATORS OF TWO VARIABLES}

Consider a third-order operator $L \in K\left[D_{1}, D_{2}\right]$. Let its symbol be $S_{1} \cdot S_{2} \cdot S_{3}$. Then, we have six factorization types when factoring into three factors,

$$
\begin{array}{lll}
\left(S_{1}\right)\left(S_{2}\right)\left(S_{3}\right), & \left(S_{1}\right)\left(S_{3}\right)\left(S_{3}\right), & \left(S_{2}\right)\left(S_{1}\right)\left(S_{3}\right), \\
\left(S_{2}\right)\left(S_{3}\right)\left(S_{1}\right), & \left(S_{3}\right)\left(S_{1}\right)\left(S_{2}\right), & \left(S_{3}\right)\left(S_{2}\right)\left(S_{1}\right),
\end{array}
$$

and six types of factorization into two factors,

$$
\begin{array}{lll}
\left(S_{1}\right)\left(S_{2} S_{3}\right), & \left(S_{2}\right)\left(S_{1} S_{3}\right), & \left(S_{3}\right)\left(S_{1} S_{2}\right), \\
\left(S_{1} S_{2}\right)\left(S_{3}\right), & \left(S_{1} S_{3}\right)\left(S_{2}\right), & \left(S_{2} S_{3}\right)\left(S_{1}\right) .
\end{array}
$$

\subsection{Two Factors}

Our theory works in the case of mutually simple factor symbols. That is, if the considered type is $\left(S_{1}\right)\left(S_{2} S_{3}\right)$, then $S_{1}$ and $S_{2} S_{3}$ must be mutually simple. Taking this into account and noting that symmetric types are considered similarly, we may confine ourselves to the following two important cases: factorization type $(X)\left(X^{2}+\right.$ $X Y)$ for the operator with the symbol $X^{2} Y+X Y^{2}$ and type $(X)\left(Y^{2}\right)$ for the operator with the symbol $X Y^{2}$.

Note that, by Theorem 7 , such ordinary obstacles may be only of orders one or zero. In the former case, an ordinary obstacle is not uniquely determined.

Theorem 10. Let

$$
\begin{gathered}
L=\widehat{\operatorname{Sym}}_{L}+a_{20} D_{x x}+a_{11} D_{x y} \\
+a_{02} D_{y y}+a_{10} D_{x}+a_{01} D_{y}+a_{00},
\end{gathered}
$$

where $a_{i j} \in K$. Let $\operatorname{Sym}_{L}=X Y(X+Y)$. Then,

$$
\begin{gathered}
\operatorname{Obst}_{(X)(Y X+Y Y)} \\
=\left(a_{02}^{2}-a_{11} a_{02}+a_{01}+\partial_{x}\left(a_{02}-a_{11}\right)\right) D_{y} \\
+a_{00}-a_{02} a_{10}+a_{02}^{2} a_{20}+2 a_{02} \partial_{x}\left(a_{20}\right) \\
-\partial_{x}\left(a_{10}\right)+a_{20} \partial_{x}\left(a_{02}\right)+\partial_{x x}\left(a_{20}\right)
\end{gathered}
$$

is an ordinary obstacle to factorization of $L$ of type $(X)(Y X+Y Y)$. Let $\operatorname{Sym}_{L}=X^{2} Y$. Then,

$$
\begin{gathered}
\operatorname{Obst}_{(Y)(X X)}=\left(a_{10}-a_{20} a_{11}-\partial_{y}\left(a_{11}\right)\right) D_{x} \\
+a_{00}-a_{20} a_{01}+a_{20}^{2} a_{02}+2 a_{20} \partial_{y}\left(a_{02}\right) \\
-\partial_{y}\left(a_{01}\right)+a_{02} \partial_{y}\left(a_{20}\right)+\partial_{y y}\left(a_{02}\right)
\end{gathered}
$$

is an ordinary obstacle to factorization of $L$ of type $(Y)(X X)$.

Proof. All factorizations of type $(X)(Y X+Y Y)$ are of the form

$$
L=\left(D_{x}+G_{0}\right) \circ\left(D_{x y}+D_{y y}+H_{1}+H_{0}\right),
$$

where $G_{0}=g_{00} \in K, H_{1}=h_{10} D_{x}+h_{01} D_{y} \in K\left[D_{x}, D_{y}\right]$, and $H_{0}=h_{00} \in K$. Comparing the second-order components on both sides of Eq. (12), we obtain the following system of linear equations in the coefficients $h_{10}, h_{01}$, and $g_{00}$ : 


$$
\left\{\begin{array}{l}
a_{20}=h_{10} \\
a_{11}=h_{01}+g_{00} \\
a_{02}=g_{00} .
\end{array}\right.
$$

Finding the only solution to the system and comparing coefficients of $D_{x}$ on both sides of (12), we obtain

$$
h_{00}=a_{10}-a_{20} a_{02}-\partial_{x}\left(a_{20}\right) \text {. }
$$

Now, we can calculate the ordinary obstacle as $P=$ $L-\left(D_{x}+G_{0}\right) \circ\left(D_{x y}+D_{y y}+H_{1}+H_{0}\right)$.

The ordinary obstacle for the factorization type $(Y)(X X)$ is found similarly.

\subsection{Three Factors}

Here, it will suffice to consider the case of a hyperbolic operator with the symbol $X Y(X+Y)$ and the factorization type $(X)(Y)(X+Y)$. In this case, an ordinary obstacle may be only of order one or zero (by Theorem 7 ) and is unique (by Theorem 8).

Theorem 11. Let

$$
\begin{aligned}
L= & D_{x} D_{y}\left(D_{x}+D_{y}\right)+a_{20} D_{x x}+a_{11} D_{x y} \\
& +a_{02} D_{y y}+a_{10} D_{x}+a_{01} D_{y}+a_{00},
\end{aligned}
$$

where $a_{i j} \in K$. The ordinary obstacle of type $(X)(Y)(X+Y)$ is given by

$$
\begin{gathered}
\operatorname{Obst}_{(X)(Y)(X+Y)} \\
=\left(a_{10}-a_{20} a_{11}-a_{20}^{2}-\partial_{x}\left(a_{20}\right)+\partial_{y}\left(s_{2}\right)\right) D_{x} \\
+\left(a_{01}-a_{02} a_{11}+a_{02}^{2}+\partial_{x}\left(-a_{11}+a_{02}\right)\right) D_{y} \\
+a_{00}+a_{20} a_{02} s_{2}+s_{2} \partial_{x}\left(a_{20}\right) \\
+\left(a_{20} \partial_{x}+\partial_{x y}+a_{02} \partial_{y}\right)\left(s_{2}\right),
\end{gathered}
$$

where $s_{2}=a_{20}-a_{11}+a_{02}$.

Proof. Let us write factorization of type $(X)(Y)(X+Y)$ in the general form as

$$
L=\left(D_{x}+g_{0}\right) \circ\left(D_{y}+h_{0}\right) \circ\left(D_{x}+D_{y}+f_{0}\right) .
$$

Comparing the second-order components on both sides of the equation, we obtain the unique solution

$$
h_{0}=a_{20}, \quad g_{0}=a_{02}, \quad f_{0}=a_{11}-a_{02}-a_{20} .
$$

Now, the ordinary obstacle can be calculated as the difference of the left-hand and right-hand sides of (13).

\section{ACKNOWLEDGMENTS}

This work was supported by the Austrian Science Foundation (FWF), project no. SFB F013/F1304.

\section{REFERENCES}

1. Grigoriev, D. and Schwarz, F., Factoring and Solving Linear Partial Differential Equations, J. Computing, 2004, vol. 73, pp. 179-197.

2. Tsarev, S.P., Generalized Laplace Transformations and Integration of Hyperbolic Systems of Linear Partial Differential Equations, Proc. ISSAC'05, 2005.

3. Kartashova, E., Hierarchy of General Invariants for Bivariate LPDOs, J. Theor. Math. Phys., 2006.

4. Shemyakova, E., A Full System of Invariants for ThirdOrder Linear Partial Differential Operators, Lecture Notes in Computer Science, Springer, 2006.

5. Shemyakova, E. and Winkler, F., Obstacle to Factorization of LPDOs, Proc. Transgressive Computing, Granada, 2006.

6. Blumberg, H., Über Algebraische Eigenschaften von Linearen Homogenen Differentialausdrücken, Diss., Göttingen, 1912.

7. www. maple.com.

8. Tsarev, S.P., On the Problem of Factorization of Linear Ordinary Differential Operators, Programming Comput. Software, 1994, vol. 20, no. 1, pp. 27-29.

9. Vinogradov, A., Krasilshchik, I., and Lychagin, B., Vvedenie $v$ geometriyu neliniinykh differentsial'nykh system (Introduction in Geometry of Nonlinear Differential Systems), Nauka, 1986. 